Au cours de la session de printemps, le Parlement a adopté la loi sur les produits thérapeutiques. La FMH salue cette décision: d'une part, le patient a le droit de renoncer à une ordonnance, ce qui garantit le libre choix dans l'obtention de médicaments. D'autre part, la nouvelle réglementation sur la transparence et la répercussion des avantages financiers permet, dans certains cas bien précis, de négocier des rabais avec l'industrie pharmaceutique dès lors que ces derniers sont transparents et n'influencent pas le choix thérapeutique. Dans son analyse, Sven Bradke, le directeur de I'APA, passe en revue l'évolution mouvementée de la révision de la LPTh.

\title{
La révision de la loi sur les pro- duits thérapeutiques est achevée!
}

\section{Sven Bradke}

Dr rer. publ. HSG, directeur de I'Association des médecins propharmaciens*

1 Communiqué officiel de la Confédération «Révision ordinaire de la loi sur les produits thérapeutiques: la procédure de consultation est ouverte du 21 octobre 2009.

* L'auteur a suivi dès le début la révision de la loi sur les produits thérapeutiques en tant que directeur de l'APA. Il a également été membre de la Taskforce qui s'est mobilisée en faveur des intérêts du corps médical dans le cadre de cette révision.

Pendant la session de printemps, le Conseil national et le Conseil des Etats ont adopté la révision de la loi sur les produits thérapeutiques, qui, initialement, prévoyait une interdiction de la propharmacie. Le débat parlementaire a duré plus de trois ans et s'est finalement concentré sur des thèmes de politique de santé comme l'accès facilité, l'homologation, la remise de médicaments ainsi que la surveillance du marché.

En octobre 2009, Pascal Couchepin, le Conseiller fédéral de l'époque, s'est présenté devant les médias pour annoncer la révision de la loi sur les produits thérapeutiques, mise sur pied en 2000 et entrée en vigueur en 2002. La révision avait pour objectif d'établir un «rapport équilibré entre régulation et dérégulation» et d'aborder des thèmes tels que la simplification de la procédure d'homologation, les avantages matériels, la remise et l'utilisation de médicaments, les principes de bonne gouvernance, l'information sur les médicaments et la surveillance du marché.

\section{De faux incitatifs?}

Ce même Conseiller fédéral a annoncé à l'époque sa volonté de supprimer la remise de médicaments par les médecins (propharmacie) afin de séparer la prescription de la remise de médicaments, et de corriger les "faux incitatifs actuels». En plus de cette interdiction, une version visant à autoriser la propharmacie "à titre exceptionnel dans les zones rurales» a également été soumise [1]. Lors de sa conférence de presse, Pascal Couchepin a en outre ajouté que la propharmacie était

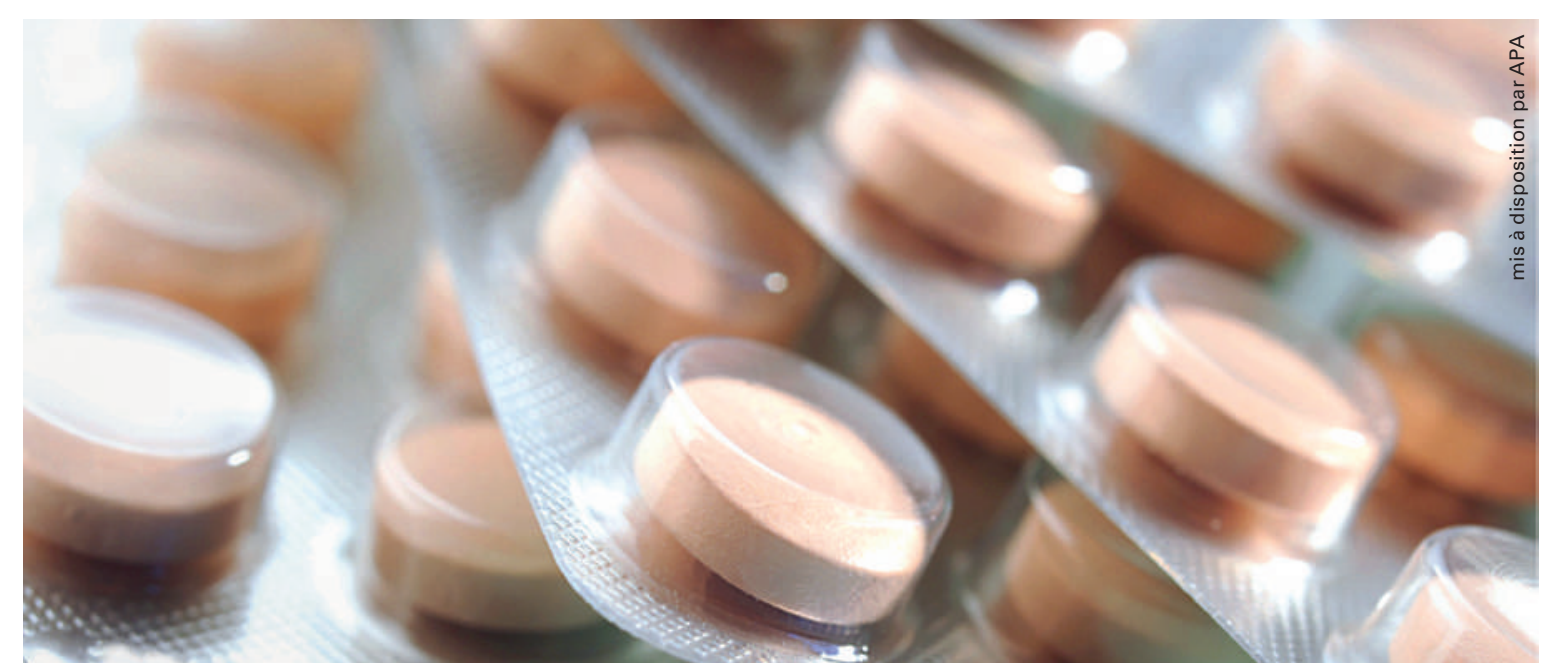

Figure 1: Le large débat politique mené au sein des commissions et des Chambres a manifestement permis d'obtenir une révision qui ne provoque aucune résistance et qui sera soumise au vote. 
onéreuse et que son interdiction constituerait également un avantage financier.

\section{Une avalanche de critiques}

Peu de temps avant son «départ à la retraite», le ministre de la santé de l'époque a donc dû faire face à une avalanche de critiques. Ses déclarations avaient suscité les plus vives réactions au sein du corps médical et dans les cantons autorisant traditionnellement la propharmacie. Le Valaisan semblait en effet insinuer sans disposer de faits concrets - que les médecins, en pratiquant la propharmacie, mettaient en danger la vie et la santé des personnes à cause de faux incitatifs et de privilèges légaux en matière de prescription et de remise de médicaments. Par ailleurs, il spéculait sur le coût de ce canal de distribution sans être en mesure de présenter des chiffres pertinents. Or, les coûts annuels de médicaments par assuré, publiés par les caissesmaladie, l'ont désavoué [2].

\section{Une argumentation téméraire}

2 Létude de Polynomics publiée en 2015 a également révélé que les déclarations faites à l'époque par le Conseiller fédéral Couchepin étaient erronées: selon létude mandatée par l'OFSP, les patients de la propharmacie génèrent des coûts de médicaments inférieurs de $13 \%$ à ceux des patients qui n'y ont pas recours. Par ailleurs, les premiers se voient plus souvent prescrire des génériques par leur médecin. Cf.l'article en allemand du BMS: Bradke S Selbstdispensation sorgt für tiefere Medikamentenkosten. Schweiz

Ärztezeitung.

2015;96(24):862-3.

3 Rapport explicatif:

Révision ordinaire de la loi sur les médicaments et les dispositifs médicaux (Loi sur les produits thérapeu tiques, LPTh), octobre

2009, p. 31

4 La Taskforce était initialement composée de représentants des médecins propharmaciens (APA), du Comité central de la FMH, de la Conférence des sociétés cantonales de méde-

cine (CCM) et de l'Association des sociétés médicales de Suisse alémanique (VEDAG).
Dans le rapport explicatif, l'argumentation avancée était la suivante: "A cause de l'asymétrie d'information entre les médecins et les patients ainsi que des incitations financières liées à la remise des médicaments, la propharmacie représente un risque sanitaire et politique» [3]. Par ailleurs, plusieurs votations populaires et des jugements rendus par des tribunaux auraient contribué à bloquer le dossier.

\section{Une propharmacie appréciée}

Tous ceux qui ont suivi le dossier savent qu'en octobre 2009, Zurich avait déjà voté trois fois en faveur de la propharmacie et que seule la recrudescence de procédures juridiques lancées par les représentants des la propharmacie aux villes de Zurich et de Winterthour. D'autres cantons n'avaient, eux aussi, enregistré jusque-là que des résultats positifs dans leur parlement respectif et dans les urnes. Cela s'explique par le fait que la propharmacie est très appréciée des patientes et des patients qui la considèrent comme une prestation du corps médical, pratique, sûre et économique.

\section{Une large opposition}

Les déclarations et les arguments fallacieux, considérés par beaucoup comme inacceptables, ont suscité une large opposition contre ce premier projet. En plus pharmaciens avait permis d'empêcher l'expansion de

\section{Qu'est-ce que I'APA?}

L'APA est I'association suisse des médecins propharmaciens. Elle défend les intérêts professionnels de ses membres dans toutes les questions liées à la remise de médicaments par les médecins. Par ailleurs, elle met à la disposition de ses membres des documents pertinents pour l'assurance qualité de la pharmacie du cabinet médical. Tout médecin possédant une autorisation pour une pharmacie de cabinet peut devenir membre ordinaire. Il est possible de s'inscrire sur le site www. patientenapotheke.ch.

de la FMH et de l'APA, nombre de sociétés de discipline médicale et de sociétés cantonales de médecine ont formulé des réponses de poids à la consultation. Les cantons alémaniques et des associations de renom ont également rejeté l'interdiction de la propharmacie. Le canton de Thurgovie a même lancé une initiative cantonale qui a ensuite été présentée à Berne.

\section{Nouveau message - sans interdiction de la propharmacie}

L’opposition véhémente, orchestrée par une taskforce du corps médical [4], avait apparemment fait forte impression. Tout au moins, le message concernant la révision de la loi sur les produits thérapeutiques, présenté par le Conseil fédéral le 7 novembre 2012, ne comportait plus de propositions d'interdiction mais se concentrait sur les thèmes suivants: les médicaments à usage pédiatrique, l'homologation simplifiée, la remise des médicaments (y compris de la médecine complémentaire), les avantages matériels, les principes de bonne gouvernance, l'information sur les médicaments et la surveillance du marché.

\section{L'ordonnance médicale: une obligation bureaucratique et onéreuse}

Une grande partie de ces thèmes a reçu le soutien du corps médical. En revanche, la résistance contre d'éventuelles limitations de la propharmacie devait continuer de s'organiser. Par exemple, l'obligation de délivrer une ordonnance également dans le cadre de la propharmacie a suscité une forte incompréhension chez les médecins en raison de la charge de travail supplémentaire qu'elle engendrerait, assortie de coûts importants d'environ 100 à 150 millions de francs par an à la charge des assurés, sans véritable contrepartie. Par ailleurs, la question de la remise par les pharmaciens de médicaments de la liste $\mathrm{B}$, en partie non soumis à l'ordonnance, ainsi que l'interdiction générale des rabais demandée à plusieurs reprises pendant le débat 
parlementaire, ont exigé un engagement politique assidu. Jusqu'à la fin, nombre de ces réglementations ont provoqué la controverse au Conseil national et au Conseil des Etats. Il a même fallu une conférence de conciliation des Chambres pour pouvoir finalement adopter la révision au cours de la dernière session. Pour l'heure, la révision de la loi sur les produits thérapeutiques, qui devrait entrer en vigueur l'année prochaine, pourrait encore être soumise à un référendum, mais à notre connaissance, aucun comité ne s'est constitué à ce jour pour recueillir des signatures.

\section{Les avantages de la révision}

Le corps médical se félicite des nombreuses précisions apportées aux termes tels que préparation originale, générique, prescription, pharmacie publique, pharmacie hospitalière et propharmacie. Nous nous réjouissons notamment de la nouvelle définition de la propharmacie, désormais ancrée dans la loi, qui n'avait pas été demandée par le Conseil fédéral. Nous saluons aussi les homologations de médicaments partiellement simplifiées. Nous approuvons également les dispositions renforcées concernant la transparence qui autorisent, à certaines conditions, le maintien des contributions de soutien pour la recherche, la formation postgraduée et continue ainsi que pour les rabais. Nous saluons aussi la création d'un nouveau registre électronique qui assurera «le recensement complet et actualisé de toutes les informations disponibles sur les médicaments autorisés en Suisse» [5].

\section{Remise de médicaments non soumis à ordonnance par les pharmacies?}

La solution trouvée, qui consiste à ce que les patients puissent renoncer à une ordonnance pour les médicaments soumis à ordonnance, que leur médecin propharmacien leur remet, constitue à nos yeux une voie praticable. C'est du moins une bien meilleure solution qu'une obligation générale de délivrer une ordonnance avec, à la clé, une lourde bureaucratie. Le corps médical continue néanmoins d'émettre des réserves concernant l'autorisation partielle des pharmaciens de remettre des médicaments soumis à ordonnance. Ceux qui ont réclamé pendant des décennies le principe du double contrôle devraient désormais être autorisés dans des cas exceptionnels justifiés, et pour des médicaments et indications spécialement désignés par le Conseil fédéral - à remettre des médicaments aux patients en toute autonomie et sans ordonnance. Le corps médical examinera avec soin cette liste que le Conseil fédéral doit établir. En effet, nous persistons à penser que les pharmaciens ne disposent pas aujourd'hui d'une formation de diagnostic médical.

\section{$4^{\mathrm{e}}$ train d'ordonnances sur les produits thérapeutiques (OPTh IV)}

Le large débat politique mené au sein des commissions et des Chambres a manifestement permis d'obtenir une révision qui ne provoque aucune résistance et qui sera soumise au vote. Tous les protagonistes - patients, industrie pharmaceutique, pharmaciens et médecins ont dû faire des concessions pour aboutir à cette proposition de compromis. Après expiration du délai référendaire, l'Office fédéral de la santé publique (OFSP) devrait présenter les nouvelles ordonnances $\left(4^{\mathrm{e}}\right.$ train d'ordonnances sur les produits thérapeutiques). Il appartiendra ensuite au corps médical d'examiner avec soin leur conformité légale et leurs répercussions.

\section{Gros plan sur les dispositions de surveillance sanitaire}

Rétrospectivement, depuis 2009, la révision de la loi sur les produits thérapeutiques s'est surtout concentrée sur des dispositions de surveillance sanitaire en laissant de côté des objectifs politiques, comme par exemple l'interdiction de la propharmacie qui était réclamée à l'époque. Cependant, de nouvelles dispositions entreront bientôt en vigueur; elles devront être présentées, expliquées et précisées au sein du corps médical. En effet, l'usage de médicaments exige des connaissances spécifiques et une procédure particulière compte tenu des risques éventuels qu'il présente pour la santé et la vie des personnes (et des animaux). La FMH et l'APA continueront donc à assurer la formation nécessaire dans les prochains mois.

\section{Teneur de quelques-uns des articles nouveaux ou modifiés de la LPTh (liste non exhaustive)}

\section{Art. 4, al. 1, let. $\mathrm{f}^{\text {bis }}$}

Prescription: décision protocolée d'un membre autorisé d'une profession médicale qui est établie conformément à l'art. 26, al. 2, pour une personne déterminée et qui confère à cette dernière un droit d'accès à des prestations médicales telles que des soins, des médicaments, des analyses ou des dispositifs médicaux;

\section{Art. 4, al. 1, let. i}

Pharmacie publique: exploitation pharmaceutique qui dispose d'une autorisation cantonale, est dirigée par 
un pharmacien, garantit des horaires d'ouverture réguliers et offre un accès direct au public;

\section{Art. 4, al. 1, let. k}

Propharmacie: remise de médicaments autorisée par le canton au sein d'un cabinet médical ou d'une institution ambulatoire de santé dont la pharmacie est placée sous la responsabilité professionnelle d'un médecin possédant une autorisation d'exercer.

\section{Art. 24, al. 1, let. a et b, et $\mathbf{1}^{\text {bis }}$}

${ }^{1}$ Sont habilités à remettre des médicaments soumis à ordonnance:

a. les pharmaciens, sur ordonnance médicale. Les pharmaciens peuvent remettre de tels médicaments sans ordonnance médicale s'ils ont un contact direct avec la personne concernée et que la remise est consignée, et:

1. s'il s'agit de médicaments et d'indications désignés par le Conseil fédéral, ou

2. dans des cas exceptionnels justifiés;

b. toute autre personne exerçant une profession médicale, conformément aux dispositions sur la propharmacie et à l'article 1, al. 3, let. c;

${ }^{1 b i s}$ Le Conseil fédéral détermine la forme et la portée de l'obligation de consigner prévue à l'al. 1, let. a.

Art. 25, al. 1, let. b, 3

${ }^{1}$ Sont habilités à remettre des médicaments non soumis à ordonnance:

c. les droguistes titulaires du diplôme fédéral;

\section{Art. 26, al. 4}

${ }^{4}$ Les personnes habilitées à prescrire et à remettre des médicaments doivent en principe délivrer une ordonnance au patient avant toute remise de médicaments à usage humain soumis à ordonnance. Le patient peut renoncer à une ordonnance.

\section{Art. 55, al. 1 et 2}

${ }^{1}$ Les personnes qui prescrivent, remettent, utilisent ou achètent à cette fin des médicaments soumis à ordonnance et les organisations qui emploient de telles personnes ne peuvent solliciter, se faire promettre ou accepter, pour elles-mêmes ou pour un tiers, un avantage indu. Il est également interdit de proposer, de promettre ou d'octroyer à ces personnes ou organisations, pour elles-mêmes ou pour un tiers, un avantage illicite.

${ }^{2} \mathrm{Ne}$ sont pas considérés comme des avantages illicites:

a. les avantages de valeur modeste et qui ont un rapport avec la pratique de la médecine ou de la pharmacie;

b. les dons destinés à la recherche, à la formation postgrade ou à la formation continue, pour autant que certains critères soient remplis;

c. les compensations accordées en contrepartie de prestations équivalentes notamment celles accordées pour les commandes et les livraisons de produits thérapeutiques;

d. les rabais ou ristournes octroyés lors de l'achat de produits thérapeutiques pour autant qu'ils n'influent pas sur le choix du traitement.

Dans le cadre de la révision de la LPTh, un article 56, al. $3^{\text {bis }}$ est introduit entre autres dans la loi fédérale du 18 mars 1994 sur l'assurance-maladie (LAMal). Il dispose de ce qui suit:

\section{Art. 56, al. $3^{\text {bis }}$}

3bis Les assureurs et les fournisseurs de prestations peuvent prévoir, dans une convention, que les avantages visés à l'alinéa 3 lettre b ne sont pas répercutés intégralement. Cette convention doit être communiquée aux autorités compétentes si celles-ci en font la demande. Elle doit prévoir la répercussion d'une majeure partie des avantages et disposer que les avantages non répercutés sont utilisés de manière vérifiable pour améliorer la qualité du traitement. 